\title{
Heredity of supraglottic exercise-induced laryngeal obstruction
}

\author{
To the Editor:
}

Respiratory symptoms on exertion, such as shortness of breath and wheezing, are commonly associated with asthma, but might also arise from the larynx [1-3]. In recent years, the emergence of exercise laryngoscopy [4] has led to a better understanding of laryngeal movement during exercise, and inspiratory supraglottic collapse on exertion has been established as a common cause of exertional breathlessness [5] that is correlated with exercise intensity [6]. Both glottic and supraglottic inspiratory closure are more commonly seen in females and most often in adolescents or young adults [7-11]. This predominance has yet to be explained; however, gender differences in larynx size/growth and consequently higher "Bernoulli forces" in females for a given respiratory demand could be a contributing factor [5]. Thus, an inherited disorder affecting laryngeal growth could also explain why the condition usually presents in adolescence $[12,13]$. A recent study by Hilland and colleagues [14] describing an association between congenital laryngomalacia and (mainly supraglottic) laryngeal closure in adolescence, points out a likely predisposition for supraglottic exercise-induced laryngeal obstruction (EILO), whereas case studies have demonstrated that congenital laryngomalacia can be inherited $[15,16]$.

A cross-sectional study (regional ethics committee no. H-3-2012-054) was undertaken to search for a hereditary component in supraglottic EILO. We examined 10 families of probands with known EILO at the supraglottic level. Participating family members, as well as the probands underwent continuous laryngoscopy during exercise. In brief, a flexible fibre optic laryngoscope was passed through the nares, following the application of a topical local anaesthetic. While wearing a safety harness, the subjects then performed a standardised incremental exercise test on a treadmill, until reaching (self-assessed) maximum effort, with the laryngoscope fastened to headgear while recording the laryngoscopic video. An experienced specialist, who did not take part in performing the tests, scored the degree of EILO as none, mild, moderate or severe, using the scoring system developed by Maat and colleagues [17]. Several studies, including our previous work and the present study classify moderate and severe grades of EILO as clinically significant, and consider mild supraglottic EILO to be a variant of normal $[3,7,9,10]$.

We found moderate (i.e. clinically significant) inspiratory supraglottic EILO in seven (24\%) of the tested family members; five females and two males (figure 1). None of the family members had severe supraglottic EILO, whereas nine (32\%) were classified as "mild". Detailed test results from probands and family members are shown in the pedigree chart (figure 1). An expert geneticist analysed the pedigree and several plausible models of inheritance indeed support the hypothesis of a hereditary component: 1) autosomal dominant, 2) X-linked dominant or 3) multifactorial. We found that an autosomal dominant model of inheritance with variable expressivity and reduced penetrance in males is the most likely of the aforementioned models, because there is at least one affected person in every generation of families in which both parents were examined, i.e. a high incidence was noted among first-degree relatives. This model would be the simplest explanation for the findings and the established gender difference. The influence of gender is a well-known phenomenon in autosomal dominant traits, for example, pre-senile baldness in which hormonal differences play a role in the expression of the trait [13]. Unknown, but similar mechanisms could be the reason for the female predominance in supraglottic EILO $[15,16]$.

Another plausible model of inheritance is X-linked dominance. These disorders usually have three characteristics. Firstly, they affect both males and females, albeit females are more often affected than males; this is a well-established trait in supraglottic EILO. Secondly, affected females can transmit the disorder to both males and females, whereas males can transmit only to females, and will do so to all of

@ERSpublications

Supraglottic EILO could be inherited. Different modes of inheritance can explain the results of this study. http://ow.ly/5XAv30csDRa

Cite this article as: Walsted ES, Hvedstrup J, Eiberg H, et al. Heredity of supraglottic exercise-induced laryngeal obstruction. Eur Respir J 2017; 50: 1700423 [https://doi.org/10.1183/13993003.00423-2017]. 


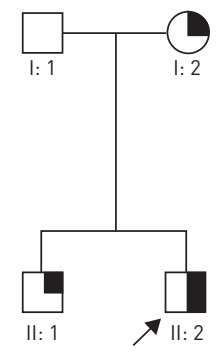

6

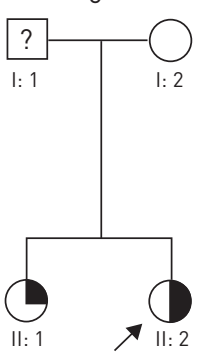

(1) Mild EILO

(1) Moderate EILO

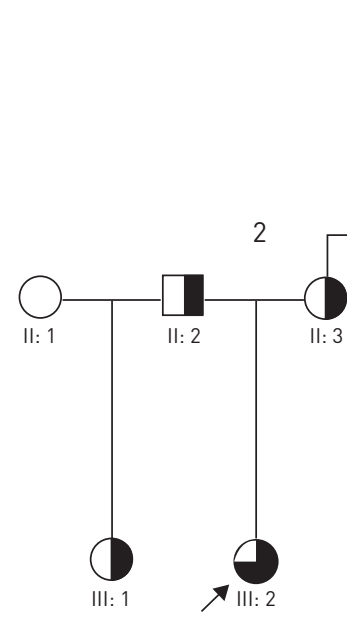

7

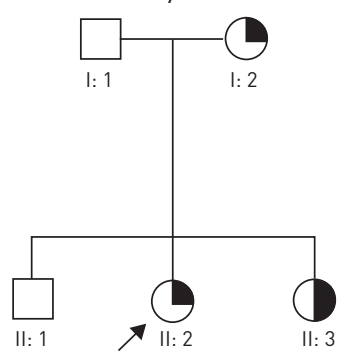

- $\square$ Severe EILO

Proband
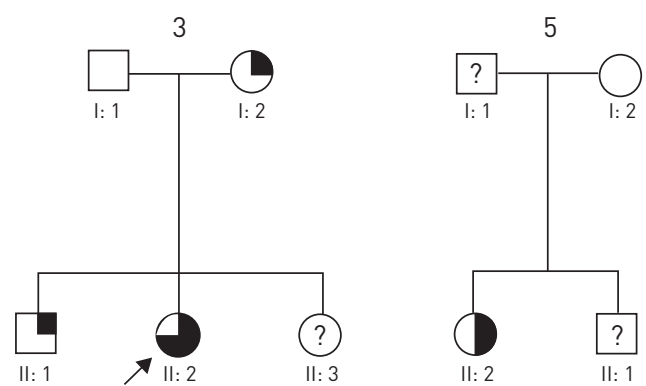

9

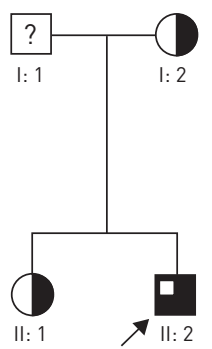

(?)

? Unknown EILO status

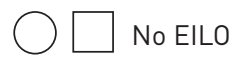

// Unknown parents
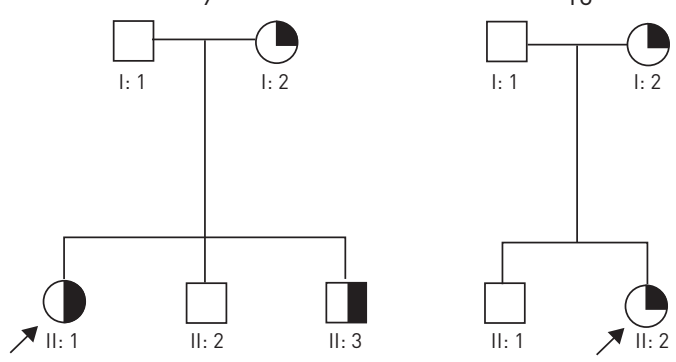

FIGURE 1 Pedigree of 10 families of probands with known exercise-induced laryngeal obstruction (EILO) at the supraglottic level.

their daughters. This feature is also supported by the data in the pedigree. Thirdly, EILO would most likely be more severe in males because of the lack of a normal X-chromosome, which is not supported by our data, nor to our knowledge by any other studies of EILO.

The findings in family 2 could also suggest a synergistic effect on the offspring, as both parents (II:2 and II:3) have the disorder. This can be explained by a model of inheritance, in which EILO is a multifactorial disorder with gender dimorphism. Such a model would also explain why females are more frequently affected. Consequently, for males to be affected, a higher genetic load is needed, compared to females, either in the form of a greater number of mutations or more severe mutations. According to this model, affected females could inherit a substantial part of their EILO from their asymptomatic and objectively disorder-free fathers. This pattern is seen in families 1, 3, 7 and 9, in which a disorder-free father and a mother with mild EILO have offspring with moderate or severe EILO. Thus, a father with severe EILO would more likely have affected daughters; a phenomenon previously described as the Carter effect [14]. The multifactorial inheritance model thus proposes that supraglottic EILO could be a spectrum disorder, inherited in the same way as height. Usually though, the incidence of multifactorial diseases in first-degree relatives is low, and they tend to have a subtle pattern of inheritance; this is in contrast with our findings that affected family members were seen in every generation, even though the families were small.

In summary, supraglottic EILO seems to run in families, and more than one genetic model can explain the findings of the present study. Given the present data, we believe the most likely model of inheritance to be an autosomal dominant disorder with variable expressivity and reduced penetrance in males. Apart from genetic predisposition, these families also share or have shared environments, which might influence the apparent accumulation of the disorder. Studies in larger families with genetic analysis could reveal the possible pathogenesis of supraglottic EILO and potentially lead to advances in diagnostics and treatment. 
Emil Schwarz Walsted $\oplus^{1,3}$, Jeppe Hvedstrup $\odot^{1,2,3}$, Hans Eiberg ${ }^{2}$ and Vibeke Backer ${ }^{1}$

${ }^{1}$ Respiratory Research Unit, Dept of Respiratory Medicine, Bispebjerg University Hospital, Copenhagen, Denmark. ${ }^{2}$ Dept of Cellular and Molecular Medicine, Panum Institute, University of Copenhagen, Copenhagen, Denmark. ${ }^{3}$ These authors contributed equally to this work.

Correspondence: Emil Schwarz Walsted, Respiratory Research Unit, Dept of Respiratory Medicine, Bispebjerg University Hospital, Bispebjerg Bakke 23, 2400 Copenhagen NV, Denmark. E-mail: emilwalsted@dadlnet.dk

Received: May 242016 | Accepted after revision: May 142017

Conflict of interest: None declared.

\section{References}

Weiss P, Rundell KW. Imitators of exercise-induced bronchoconstriction. Allergy Asthma Clin Immunol 2009; 5: 7. Hull JH. Not all wheeze is asthma: time for patients to exercise their rights. Thorax 2015; 70: 7-8.

Johansson $\mathrm{H}$, Norlander $\mathrm{K}$, Berglund $\mathrm{L}$, et al. Prevalence of exercise-induced bronchoconstriction and exercise-induced laryngeal obstruction in a general adolescent population. Thorax 2015; 70: 57-63.

4 Heimdal J-H, Roksund OD, Halvorsen T, et al. Continuous laryngoscopy exercise test: a method for visualizing laryngeal dysfunction during exercise. Laryngoscope 2006; 116: 52-57.

5 Roksund OD, Heimdal $\mathrm{J}-\mathrm{H}$, Clemm $\mathrm{H}$, et al. Exercise inducible laryngeal obstruction: diagnostics and management. Paediatr Respir Rev 2017; 21: 86-94.

6 Olin JT, Clary MS, Fan EM, et al. Continuous laryngoscopy quantitates laryngeal behaviour in exercise and recovery. Eur Respir J 2016; 48: 1192-1200.

7 Walsted ES, Hull JH, Sverrild A, et al. Bronchial provocation testing does not detect exercise-induced laryngeal obstruction. J Asthma 2017; 54: 77-83.

8 Marcinow AM, Thompson J, Chiang T, et al. Paradoxical vocal fold motion disorder in the elite athlete: experience at a large division I university. Laryngoscope 2014; 124: 1425-1430.

9 Nielsen EW, Hull JH, Backer V. High prevalence of exercise-induced laryngeal obstruction in athletes. Med Sci Sports Exerc 2013; 45: 2030-2035.

10 Christensen PM, Thomsen SF, Rasmussen N, et al. Exercise-induced laryngeal obstructions: prevalence and symptoms in the general public. Eur Arch Otorhinolaryngol 2011; 268: 1313-1319.

11 Røksund OD, Maat RC, Heimdal J-H, et al. Exercise induced dyspnea in the young. Larynx as the bottleneck of the airways. Respir Med 2009; 103: 1911-1918.

12 Castelli WA, Ramirez PC, Nasjleti CE. Linear growth study of the pharyngeal cavity. J Dent Res 1973; 52: $1245-1248$.

13 Wysocki J, Kielska E, Orszulak P, et al. Measurements of pre- and postpubertal human larynx: a cadaver study. Surg Radiol Anat 2008; 30: 191-199.

14 Hilland M, Roksund OD, Sandvik L, et al. Congenital laryngomalacia is related to exercise-induced laryngeal obstruction in adolescence. Arch Dis Child 2016; 101: 443-448.

15 Chen JL, Messner AH, Chang KW. Familial laryngomalacia in two siblings with syndromic features. Int J Pediatr Otorhinolaryngol 2006; 70: 1651-1655.

16 Shohat M, Sivan Y, Taub E, et al. Autosomal dominant congenital laryngomalacia. Am J Med Genet 1992; 42: 813-814.

17 Maat RC, Røksund OD, Halvorsen T, et al. Audiovisual assessment of exercise-induced laryngeal obstruction: reliability and validity of observations. Eur Arch Otorhinolaryngol 2009; 266: 1929-1936. 